\title{
IoT with Evolutionary Algorithm Based Deep Learning for Smart Irrigation System
}

\author{
P. Suresh ${ }^{1, *}$, R. H. Aswathy ${ }^{1}$, Sridevi Arumugam ${ }^{2}$, Amani Abdulrahman Albraikan ${ }^{3}$, \\ Fahd N. Al-Wesabi ${ }^{4}$, Anwer Mustafa Hilal $^{5}$ and Mohammad Alamgeer ${ }^{6}$
}

\author{
${ }^{1}$ Department of Computer Science and Engineering, KPR Institute of Engineering and Technology, Coimbatore, \\ 641407, India \\ ${ }^{2}$ Department of Electrical Sciences, Adithya Institute of Technology, Coimbatore, 641107, India \\ ${ }^{3}$ Department of Computer Science, College of Computer and Information Sciences, Princess Nourah Bint Abdulrahman \\ University, Saudi Arabia \\ ${ }^{4}$ Department of Computer Science, King Khalid University, Muhayel Aseer, Saudi Arabia \& Faculty of Computer and IT, \\ Sana'a University, Sana'a, Yemen \\ ${ }^{5}$ Department of Computer and Self Development, Preparatory Year Deanship, Prince Sattam Bin Abdulaziz University, \\ Alkharj, Saudi Arabia \\ ${ }^{6}$ Department of Information Systems, King Khalid University, Muhayel Aseer, Saudi Arabia \\ *Corresponding Author: P. Suresh. Email: sps1765@gmail.com \\ Received: 14 July 2021; Accepted: 16 August 2021
}

\begin{abstract}
In India, water wastage in agricultural fields becomes a challenging issue and it is needed to minimize the loss of water in the irrigation process. Since the conventional irrigation system needs massive quantity of water utilization, a smart irrigation system can be designed with the help of recent technologies such as machine learning (ML) and the Internet of Things (IoT). With this motivation, this paper designs a novel IoT enabled deep learning enabled smart irrigation system (IoTDL-SIS) technique. The goal of the IoTDL-SIS technique focuses on the design of smart irrigation techniques for effectual water utilization with less human interventions. The proposed IoTDL-SIS technique involves distinct sensors namely soil moisture, temperature, air temperature, and humidity for data acquisition purposes. The sensor data are transmitted to the Arduino module which then transmits the sensor data to the cloud server for further process. The cloud server performs the data analysis process using three distinct processes namely regression, clustering, and binary classification. Firstly, deep support vector machine (DSVM) based regression is employed was utilized for predicting the soil and environmental parameters in advances such as atmospheric pressure, precipitation, solar radiation, and wind speed. Secondly, these estimated outcomes are fed into the clustering technique to minimize the predicted error. Thirdly, Artificial Immune Optimization Algorithm (AIOA) with deep belief network (DBN) model receives the clustering data with the estimated weather data as input and performs classification process. A detailed experimental results analysis demonstrated the promising performance of the presented technique over the other recent state of art techniques with the higher accuracy of 0.971 .
\end{abstract}


Keywords: Smart irrigation; precision agriculture; internet of things; deep learning; machine learning

\section{Introduction}

Water is one of the essential factors limiting agriculture. Such factors are increased in the areas where there is scarcity of water resources [1]. In such areas, the significance of effectively handling irrigation is an essential factor for sustainable production. It comprises agriculture methods which have allowed for optimizing irrigation management, by using drip irrigation system for controlled deficit irrigation approaches that are capable of maintaining crops with low irrigation volume. Information and communication technologies (ICT) have been provided to sustainably managing of water in agricultural production [2]. The placement of wireless sensor networks in yields via Internet of Things (IoT) techniques and the remote management of data using $\mathrm{CC}$ have enabled huge monitoring of agriculture parameters that produce huge number of data [3]. But, the modern continuity of irrigation system needs to perform tools which enable automatic scheduling of irrigation. It should involve sensor nodes to offer distinct variables [4].

Conventionally, such variables are interrelated to environment conditions and offer data regarding the entire crop water conditions utilizing climate station and the soils water condition or volumetric content that shows the availability of water for the plant [5]. The most generally utilized soil parameter sensor nodes are using dielectric properties, as they are flexible and cheap, though it's effective functioning needs complicated standardization, considering the aspects like spatial variability of the soil state, temperature, water salinity, soil structure, and texture [6]. Various sensor nodes like satellites, infrared radiometers (IR) multispectral, and thermal cameras, are utilized for estimating the water crop requirements. About irrigation automation, soil sensor nodes had begun to be utilized for these purposes, taking into account volumetric water content and thermal sensors, water matric potential, and, currently, their integration with wireless techniques for flexible execution [7]. But, irrigation management could also consider more parameters, comprising water quality, hydro-physical property of particular soil, their stages of development, factors related to productivity, and the parameter interrelated to other crops, the implementation of deficit irrigation strategies, and fruit quality that prevents effective management of irrigation threshold.

System depending upon machine learning (ML) methods utilize the prior irrigation management experience of human experts for training a scheme to regenerate that expert's behaviour. Fuzzy logic (FL), artificial neural networks (ANNs), or regression processes were utilized newly for managing automated irrigation [8].

This paper designs a novel IoT enabled deep learning enabled smart irrigation system (IoTDLSIS) technique. The proposed IoTDL-SIS technique involves dissimilar sensors such as soil moisture, temperature, air temperature, and humidity for data acquisition purposes. The sensor data are transferred to the Arduino module which then transmits the sensor data to cloud server for further process. The cloud sever accomplishes the data analysis process by means of three different processes namely deep support vector machine (DSVM) based regression, clustering, and Artificial Immune Optimization Algorithm (AIOA) with deep belief network (DBN) based classification. A detailed experimental results analysis outperformed the promising efficiency of the presented technique over the other recent state of art techniques interms of different models. 


\section{Related Works}

Munir et al. [9] used a smart method efficiently qualified to utilize ontology for making $50 \%$ of the decision, and another $50 \%$ of the decision based on the sensor data values. Furthermore, an edge server is presented among the GSM module and main IoT server. This technique would evade the overload of the IoT server for reducing the latency rate and for processing data. Torres-Sanchez et al. [10] intended to carry out research of various learning methods for determining the goodness and error comparative to expert's decisions. The 9 orchards have been verified in the course of 2018 by LR, RFR, and SVR approaches as the engine of IDSS projected.

Bhoi et al. [11], proposed an IoT enabled ML trained proposal scheme for using effective water with the nominal interference of the farmer. IoT device is placed in the crop fields for accurately collecting the environmental and ground information's. In order to create the scheme more adaptive and robust, an in-built feedback method is included for this proposal scheme. Yu et al. [12] improving the accuracy of forecasting SWC at multiple-depth, the ResBiLSTM method has been projected, where SWC data and continuous meteorological have been transformed and gridded as module input, and later time sequence and higher dimension spatial features have been extracted with BiLSTM and ResNet, correspondingly, and incorporated with a meta learner. Kashyap et al. [13] proposed a DL NN based IoT enabled smart irrigation scheme for accurate agriculture (DLiSA). This is a feedback incorporated scheme which saves its better efficiency in the weather of other regions for a time period.

Akshay et al. [14] proposed an innovative technique on the basis of intelligent scheme for predicting the irrigation needs of a region by sensing ground parameters like water level, moisture of the soil, and temperature humidity with the help of ML method. Present techniques such as Kmean and SVM are confronting overfitting issues for overcoming these problems i.e., utilizing KNN approach as well as this projected scheme has a capability for realizing a fully automatic irrigation system and deliberated in detail regarding the data processing result with 3 weeks predetermined data depends on the presented method. Karar et al. [15] goals at storing the wasted water in the procedure of irrigation utilizing IoT depend on a group of MLP-NN and sensor nodes. The sensor measures the environmental aspects; like soil moisture, temperature, and humidity for estimating the needed time for the process of water irrigation.

Krishnan et al. [16] proposed a smart irrigation scheme which assists farmers to water their agricultural fields with the help of GSM transmission. This method offers acknowledgment messages regarding the jobs status like temperature of surrounding environment, condition of motor about a main solar power/power supply and humidity level of soil. FL controller is utilized for computing input variables (for example temperature, humidity, and soil moisture) and for producing outputs of motor conditions. Furthermore, the scheme also turns off the motor for saving power if there is an availableness of rain as well as prevent the crops utilizing panels from unconditioned rain. In Nawandar et al. [17], a sample crop test bed was selected for presenting the outcomes of the projected organism, which includes remote data viewing, neural net decision making, and irrigation schedule. The NN offers essential knowledge to the device which considers present sensor input and masks the irrigation schedule for effective irrigation.

Decision support systems (DSSs) are ML application which utilize the proficiency of an agronomist (human experts) for learning irrigation scheduling pattern and simulate human activity for making decisions. Moreover, DSS permits a continuous learning procedure (when utilized) and adopt their efficiency to different objectives/context changes. Thus, DSS in agriculture are effective tools for managing optimum irrigation and have shown optimum behaviour. Such schemes have been implemented and defined for a number of years in the agriculture sectors for huge range of 
applications, also to develop crop growth models, agricultural management models, financial and irrigation management.

\section{The Proposed Model}

Fig. 1 demonstrates the working process of proposed IoTDL-SIS method. In this study, a smart irrigation system using IoTDL-SIS technique is designed to reduce the amount of water utilization in precision agriculture. The proposed IoTDL-SIS technique operates on three levels namely farmland, cloud server, and client level. At the first farmland level, the IoT sensors are placed in the farmland to gather details and sent to Arduino for data transmission into the cloud server. Secondly, the proposed model gets executed at the cloud server level to determine the need for water irrigation. Lastly, at the client level, the predicted information is sent to the client and necessary operations were taken.

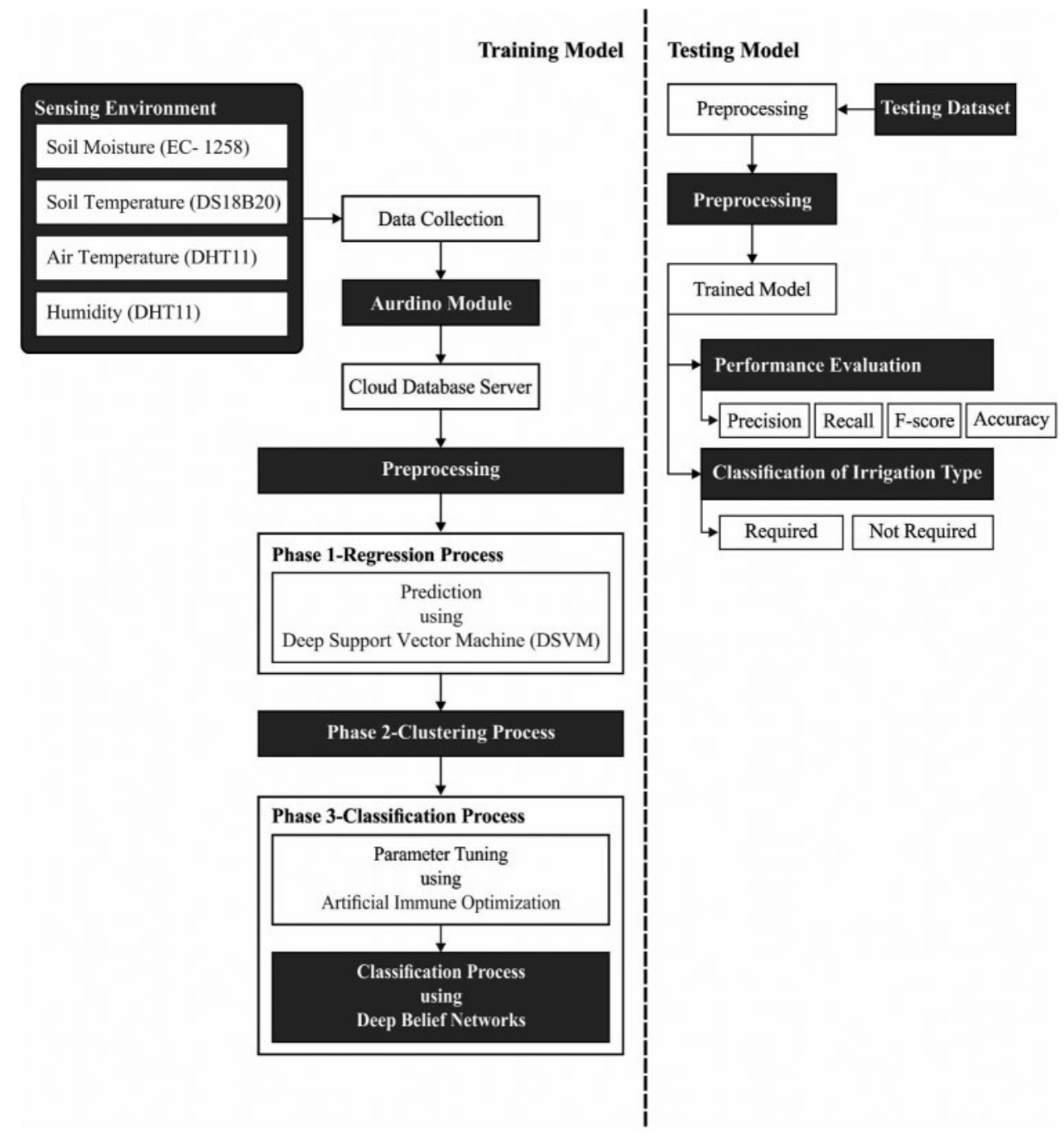

Figure 1: Working process of IoTDL-SIS method 


\subsection{Farmland Level}

The primary one is the crop field stage in which distinct sensor nodes are placed in the region. Different sensor nodes such as soil temperature (DS18B20), soil moisture (EC-1258), humidity (DHT11), and air temperature (DHT11), are utilized for gathering these environmental and soil characteristics. Each sensor nodes gather information and transmit it to the Arduino. Later, Arduino forwards the sensor data to the cloud server. Each sensor data are gathered two times daily and forward to cloud storage with the help of microcontroller device. The average value is estimated and saved as the last readings for the specific day. In order to remove the interdependency between the utilized variables, the Pearson relation is calculated. It is established that there is no stronger relation present. Now, they utilize the Arduino microcontroller since it needs lower energy.

\subsection{Cloud Server Level}

The next stage is the cloud level in which the cloud server is utilized for providing service to the user. This ML unit is the core of these smart systems that has 2 parts. (i) Regression method i.e., used for predicting the environment and soil variables beforehand. As a result, it could be utilized efficiently for improving the efficiency of the scheme. The variables i.e., considering from the predicted weather data consists of precipitation, atmospheric pressure, wind speed, and solar radiation. Such forecasted values are pass by a clustering method for reducing the forecasted errors. Another ML based models take the result of clustering method and predicted weather data as input. This binary classification method classifies the forecasted instance into 2 predetermined groups: irrigation required (Y) or not required (N). The result of this ML model is saved in the database for upcoming activities. Depending upon the proposal of ML method, the controller would transmit irrigation suggestions to the user through the Android application. According to the agriculture survey, the equation utilized to calculate the water necessity is shown in Eq. (1).

$E V_{o} * C_{f} 1 / 4=W_{\text {need }}$

Whereas, $E V_{o}=$ rate of evaporation $C_{f}=$ crop factor $W_{\text {need }}=$ quantity of water required.

\subsection{DSVM Model for Regression Process}

DSVM is a new technique that utilizes SVMs for learning to remove higher level features from input vectors, next to that these features are provided as essential SVM to do actual forecast of the farmland parameters. The entire model was trained with east gradient ascent and descent learning techniques on the dual objective of essential SVM. An essential SVM learns for maximizing this objective, but the feature layer SVM is learned for minimizing it. Rather than adopting some kernel weight, it can utilize huge DSVM frameworks, sometimes containing hundred SVM in the initial layer. Still, the difficulty of DSVM scale only linearly with amount of SVM related to the classic SVM. Also, the strong regularization power of an essential SVM avoids overfitting.

It can be utilized regression dataset: $\left\{\left(x_{1}, y_{1}\right), \ldots,\left(x_{\ell}, y_{\ell}\right)\right\}$ where $x_{i}$ represents the input vector and $y_{i}$ implies the target output. The framework of 2 layers of DSVM is demonstrated in Fig. 2. Initial, it has input layer of $D$ input. Afterward, an entire of $d$ pseudo-randomly established SVMs $S_{a}$, every one learned for extracting one feature $f(x)_{a}$ from input patterns $x$. Lastly, an essential SVM $M$ which 
estimates the target function utilizing the removed feature vector as input. To calculating the featurelayer signified $f(x)$ of input vector $x$, it can be utilized:

$f(x)_{a}=\sum_{i=1}^{\ell}\left(\alpha_{i}^{*}(a)-\alpha_{i}(a)\right) K\left(x_{i}, x\right)+b_{a}$

which iteratively estimates all elements $f(x)_{a}$. In this formula, $\alpha_{i}^{*}(a)$ and $\alpha_{i}(a)$ are SVM coefficients for SVM $S_{a}, b_{a}$ is their bias, and $K$ implies the kernel function [18]. To compute the output of entire model, it can be utilized:

$g(f(x))=\sum_{i=1}^{\ell}\left(\alpha_{i}^{*}-\alpha_{i}\right) K\left(f\left(x_{i}\right), f(x)\right)+b$

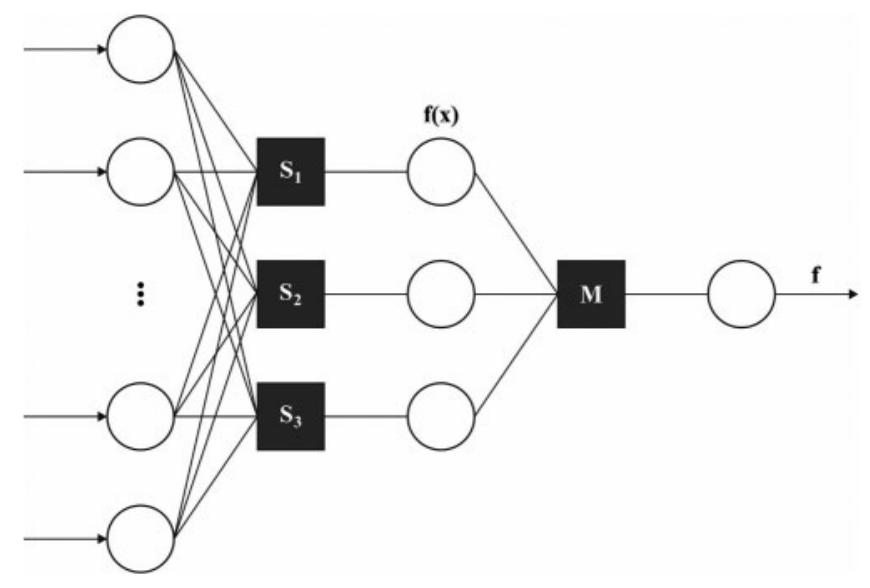

Figure 2: Structure of DSVM

Learning Algorithm. The learning technique alters the SVM coefficient of every SVM with minmax formulation of dual objectives $W$ of essential SVM:

$$
\begin{aligned}
& \min _{\mathrm{f}(\mathrm{x})} \max _{\alpha, \alpha^{*}} W\left(f(x), \alpha^{(*)}\right)=-\in \sum_{i=1}^{\ell}\left(\alpha_{i}^{*}+\alpha_{i}\right)+\sum_{i=1}^{\ell}\left(\alpha_{i}^{*}-\alpha_{i}\right) y_{i} \\
& -\frac{1}{2} \sum_{i, j=1}^{\ell}\left(\alpha_{i}^{*}-\alpha_{i}\right)\left(\alpha_{j}^{*}-\alpha_{j}\right) K\left(f\left(x_{i}\right), f\left(x_{j}\right)\right)
\end{aligned}
$$

It can be made an easy gradient ascent technique for training the SVM. This technique adopts the SVM coefficient $\alpha^{(*)}$ (referring each $\alpha_{i}^{*}$ and $\alpha_{i}$ ) near a (local) maximal of $W$, where $\lambda$ represents the learning rate: $\alpha_{i}^{(*)} \leftarrow \alpha_{i}^{(*)}+\lambda \cdot \partial W / \partial \alpha_{i}^{(*)}$ The resulting gradient ascent learned rule for $\alpha_{i}$ is:

$\alpha_{i}=\alpha_{i}+\lambda\left(-\varepsilon-y_{i}+\sum_{j}\left(\alpha_{j}^{*}-\alpha_{j}\right) K\left(f\left(x_{i}\right), f\left(x_{j}\right)\right)\right)$ 
It can utilize radial basis function (RBF) kernel in both layers of 2 layered DSVM. The outcomes with other kernels are least. An essential SVM:

$K\left(f\left(x_{i}\right), f(x)\right)=\exp \left(-\sum_{a} \frac{\left(f\left(x_{i}\right)_{a}-f(x)_{a}\right)^{2}}{\sigma_{m}}\right)$

The system creates novel datasets to all feature-layer SVM $S_{a}$ with BP-like approach to creating samples: $\left(x_{i}, f\left(x_{i}\right)_{a}-\mu \cdot \delta W / \delta f\left(x_{i}\right)_{a}\right)$, where $\mu$ implies the few learning rates, and $\delta W / \delta f\left(x_{i}\right)_{a}$ is provided as:

$\frac{\delta W}{\delta f\left(x_{i}\right)_{a}}=\left(\alpha_{i}^{*}-\alpha_{i}\right) \sum_{j=1}^{\ell}\left(\alpha_{j}^{*}-\alpha_{j}\right) \frac{f\left(x_{i}\right)_{a}-f\left(x_{j}\right)_{a}}{\sigma_{m}} \cdot K\left(f\left(x_{i}\right), f\left(x_{j}\right)\right)$

The feature removing SVM is pseudo-randomly established and after alternate training of essential SVM and feature layer SVM are implemented the amount of epochs. The bias value is calculated from average error.

\subsection{DBN Model for Classification Process}

In order to carry out smart irrigation, DBN method is employed using 3 phases such as updating, fine-tuning and pre-training phases. In general, RNN is mainly used to predict the time sequence data testing. Basically, NN using different layers is defined as Deep Neural Network (DNN) [19]. The appropriate technique of DNN depends on Back Propagation (BP) i.e., highly complicated. Numerous learning techniques equal to DNN like drop out, pre-training has been made. There are several approaches of DNN namely CNN, DBN, etc. In DBN, it could be arranged by several RBMs, and it is performed a training method layer wise to overcome this problem. As well, the CNN training method isn't fit for multilayer trained networks. The overall training function of DBN has been categorized as tuning and pre-training phases.

\subsubsection{Pre-Training Stage}

As a result, that it could be deep structure and substantial feed forward network, the DBN was applied. The instances are employed from input to the resulting layer using large hidden layers with additional layers. Based on the DBN technique and the hidden unit classifiers the approach themselves produces activation purpose. The precise unit $v$ is employed to the function of training RBM vector is expressed in Eq. (8).

$F(v, h)=-\sum_{p=1}^{P} \sum_{q=1}^{Q} W_{p q} v_{p} h_{q}-\sum_{p=1}^{P} \alpha_{p} v_{p}-\sum_{q=1}^{Q} \beta_{q} h_{q}$

The maximization of Eq. (8) is provided below. $\mathrm{W}_{\mathrm{pq}}$ indicates the programmatic interaction amongst visible unit $\mathrm{v}_{\mathrm{p}} \&$ hidden unit $\mathrm{h}_{\mathrm{q}}, \alpha, \beta$ denoted as bias term, $P, Q$ represents the number of hidden and visible units. The following log probability of a training vector considers weights of inconsistency. If the hidden unit is from RBM, it doesn't provide a direct response which assists to attain an optimum impartial instance from $\left(\mathrm{V}_{\mathrm{p}}, \mathrm{h}_{\mathrm{q}}\right)_{\text {data }}$ 
$\rho\left(h_{q}=1 \mid v\right)=\delta\left(\sum_{q=1}^{Q} W_{p q} v_{p}+\alpha_{q}\right)$

Wheres $\delta(x)$ denotes the logistic sigmoid function. $\frac{1}{(1+\exp (x))}, v_{p}, h_{q}$ indicates the unbiased sample.

\subsubsection{Updating Process}

It process a complicated phase is provided below.

$\Delta W_{p q} \theta\left(v_{p} h_{q}\right)_{\text {data }}-\left(v_{p} h_{q}\right)_{\text {reconstruction }}$

Here, RBM is trained well. The different RBM is arranged upon a frame used of multilayer models. Also, a unique RBM could be determined that has been arranged. When the input visible layer is arranged as a vector, the qualities to units are created efficiently along with RBM layers and make use of shared methods in present biases and weights. Therefore, the last layer undergoes training separately and is locked in a new RBM. The attained DNN weights are embedded with the fine-tuning phase.

\subsubsection{Fine Tuning Phase}

The procedure is executed based on BP technique. In order to categorize the medical images into two phases, the resulting layer is arranged from top of DNN. Further, $N$ amount of input neurons and the hidden layers were used in DL method. As a result, the low error value was estimated; and high accuracy of DL classification would be attained by optimized weights.

\subsection{AIOA Based Hyperparameter Optimization of DBN Model}

In order to effectually tune the hyperparameters of the DBN model, the AIOA is employed. Artificial immune algorithm operators contain: affinity estimation function, operator calculation of separate levels, incentive meter computes son, immune selective function, clone (separate multiplication), mutation operator, operator, and population suppression cloning Refresh operator. In genetic algorithm (GA) and other same intelligence optimization techniques, artificial immune algorithm for relying on evolutionary optimized model function for achieving. As the encoded technique is a real number coding, discrete coding, different coding approaches in the operator are distinct.

Antibody density is generally determined as:

$\operatorname{den}\left(A_{i}\right)=\frac{1}{m} \sum_{i=1}^{m} \operatorname{aff}\left(A_{i}, B_{j}\right)$

where $A_{i}$ implies the initial $i$-species antibody; $m$ represents the population sizes; aff $\left(A_{i}, A_{j}\right)$ for the antibody $i$ and $j$ in affinity antibody. Estimation of antibody density is prerequisite for antibodydefinition of antibody affinity which aff $(u, v): S \times S \rightarrow R$, where $S$ signifies the possible solution regions [20]. Antibody- antibody affinity mostly dependent upon computation of antibodyantigen affinity computation technique dependent upon Euclidean distance was computed depends on Hamming distance computation technique dependent upon information entropy computation techniques. 
(1) Euclidean distance-based antibody-antibody affinity computation

In order to real coded technique, antibody - antibody affinity antibodies are frequently Euclidean distance amongst vectors are estimated.

(2) According to antibody-antigen affinity of antibodies - antibody affinity estimation

All individuals antibody is affinity for anigen aff (x), thus antibodies - antibody affinity as antigenantibody affinity for defining.

aff $\left(A_{i}, B_{j}\right)=\left\{\begin{array}{l}1, \operatorname{aff}\left(A_{i}\right)=\operatorname{aff}\left(B_{j}\right), \\ \left(1+\left(\operatorname{aff}\left(A_{i}\right)-\operatorname{aff}\left(B_{J}\right)\right)^{2}\right)^{-1}, \\ \operatorname{aff}\left(A_{i}\right) \neq \operatorname{aff}\left(B_{J}\right) .\end{array}\right.$

Affinity estimation and problems definitely compared with various optimization issues are real problems in understanding the premise based on features of issue explanation of affinity estimation functions. The affinity characterization of immune cell and antigen binding strength, and fitness in GA same. The affinity estimation function was generally a function aff $(x): S \rightarrow R$, where $S$ refers the possible solution region, $R$ implies the real number fields. The input is function of individual's antibodies (possible solution), the output is affinity of estimation outcomes. The stocks refresh function ( $T d: S \rightarrow S$ ) of the population of antibodies is minimum incentive to refresh, eliminate them from antibody population arbitrarily created antibody, and the novel antibody for replacing, is conducive for maintaining the diversity of antibody, and discover novel possible solution space area.

The mutation operator ( $T m: S \rightarrow S$ ) on the clone operator gained the outcomes of antibody as to the cloning. The line mutation function for generating affinity mutation, for achieving local search. The mutation function was artificial immune algorithm is the potential for generating novel antibody, an essential of regional search function, the efficiency of technique greatly. In the clone operator $(T c: S \rightarrow S$ ), immune election operator is elected for replicating the separate antibody.

\subsection{Client Level}

In this phase, the user interact with Android application for entering the data regarding the crop and farmer. Farmer's credential is utilized for authenticating the user via login process. In crop detail, the farmer might need to present the data by choosing the drop down menus such as a crop name, session, date of sowing, total crop days, etc. By this application, the farmer might receive data related to the field and crop. Therefore, on getting the irrigation and sensor data suggestions on the Android application, the user might command the on or off to order the microcontroller. Therefore, they have an automated irrigation scheme, i.e., utilized for increasing the efficiency of the crop by supplying an optimum quantity of water.

\section{Performance Validation}

This section validates the performance of the presented technique against benchmark dataset. The instances that exist in the dataset are partitioned into two classes namely class 1: irrigation required (soil moisture $<=60$ and temperature $>=25$ and humidity $<=60$ ) and class 2 : irrigation not required (soil moisture $>=61$ and temperature $<25$ and humidity $>=61$ ). 

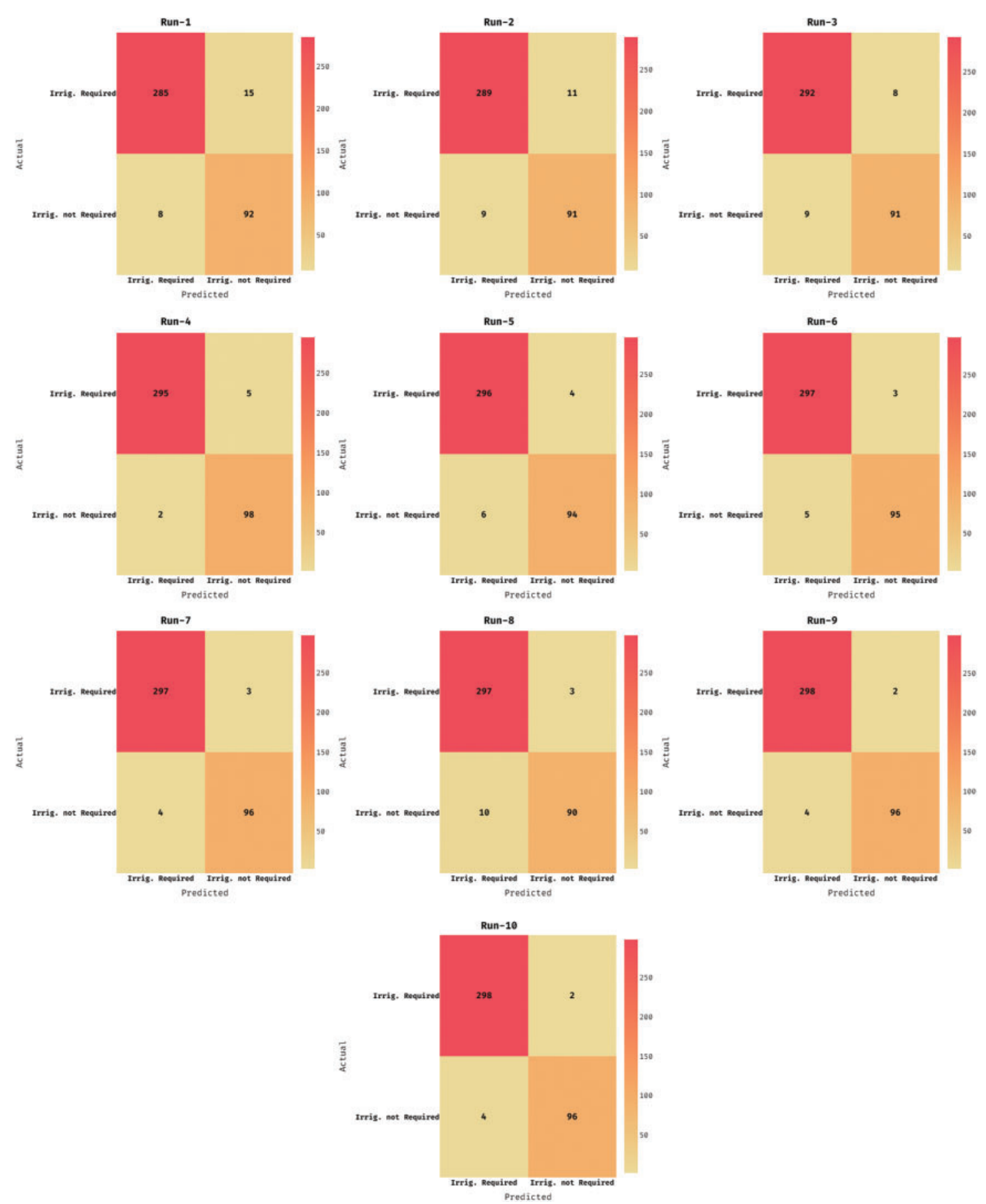

Figure 3: Confusion matrix of IoTDL-SIS method with distinct runs

Fig. 3 shows the set of confusion matrices generated by the IoTDL-SIS method on the smart irrigation prediction. The figure demonstrated that the IoTDL-SIS method has accomplished maximum performance under distinct runs of execution. For instance, with run-1, the IoTDL-SIS method has 
classified a set of 285 instances into IR and 92 instances into INR. Similarly, with run-3, the IoTDLSIS methodology has classified a set of 292 instances into IR and 91 instances into INR. Likewise, with run-5, the IoTDL-SIS approach has classified a set of 296 instances into IR and 94 instances into INR. Additionally, with run-7, the IoTDL-SIS technique has classified a set of 297 instances into IR and 96 instances into INR. Lastly, with run-10, the IoTDL-SIS algorithm has classified a set of 298 instances into IR and 96 instances into INR.

A brief results analysis of the IoTDL-SIS technique is examined under distinct runs in Tab. 1 and Fig. 4. The experimental results demonstrated that the IoTDL-SIS technique has been found to be an effective classification tool under distinct runs. For instance, with run-1, the IoTDL-SIS technique has gained a precision of 0.973 , recall of 0.950 , accuracy of 0.943 , and F-score of 0.961 . Also, with run-2, the IoTDL-SIS manner has increased precision of 0.970 , recall of 0.963 , accuracy of 0.950 , and F-score of 0.967 . Followed by, with run-3, the IoTDL-SIS approach has attained a precision of 0.970 , recall of 0.973 , accuracy of 0.958 , and F-score of 0.972 . Additionally, with run-4, the IoTDLSIS methodology has reached a precision of 0.993 , recall of 0.983 , accuracy of 0.983 , and F-score of 0.988. Simultaneously, with run-5, the IoTDL-SIS technique has obtained a precision of 0.980 , recall of 0.987 , accuracy of 0.975 , and F-score of 0.983 . Concurrently, with run-6, the IoTDL-SIS algorithm has achieved a precision of 0.983 , recall of 0.990 , accuracy of 0.980 , and F-score of 0.987 . Next, with run-7, the IoTDL-SIS method has obtained a precision of 0.987 , recall of 0.990 , accuracy of 0.968 , and F-score of 0.988. Afterward, with run-8, the IoTDL-SIS technique has gained a precision of 0.967, recall of 0.990 , accuracy of 0.968 , and F-score of 0.979 . Also, with run-9, the IoTDL-SIS technique has gained a precision of 0.987 , recall of 0.993 , accuracy of 0.985 , and F-score of 0.990 . Lastly, with run-10, the IoTDL-SIS methodology has reached a precision of 0.987 , recall of 0.993 , accuracy of 0.985 , and F-score of 0.990 .

Table 1: Results analysis of various runs on proposed IoTDL-SIS method with respect to different measures

\begin{tabular}{lllll}
\hline No. of runs & Precision & Recall & Accuracy & F-score \\
\hline Run-1 & 0.973 & 0.950 & 0.943 & 0.961 \\
Run-2 & 0.970 & 0.963 & 0.950 & 0.967 \\
Run-3 & 0.970 & 0.973 & 0.958 & 0.972 \\
Run-4 & 0.993 & 0.983 & 0.983 & 0.988 \\
Run-5 & 0.980 & 0.987 & 0.975 & 0.983 \\
Run-6 & 0.983 & 0.990 & 0.980 & 0.987 \\
Run-7 & 0.987 & 0.990 & 0.983 & 0.988 \\
Run-8 & 0.967 & 0.990 & 0.968 & 0.979 \\
Run-9 & 0.987 & 0.993 & 0.985 & 0.990 \\
Run-10 & 0.987 & 0.993 & 0.985 & 0.990 \\
Average & 0.980 & 0.981 & 0.971 & 0.980 \\
\hline
\end{tabular}

Finally, a detailed comparative analysis of the IoTDL-SIS technique with existing techniques takes place in Tab. 2 and Figs. 5 and 6. By examining the performance of the IoTDL-SIS technique on the applied dataset-1 with (70:30) Training and Testing, the NB model has shown inferior outcome with the least accuracy of 0.835 , precision of 0.827 , recall of 0.81 , and F1-score of 0.818 . Followed by, the DT (C4.5) technique has attained slightly improved results with an accuracy of 0.857 , precision of 0.851 , 
recall of 0.838 , and F1-score of 0.845 . Then, the SVM model has depicted moderate performance with an accuracy of 0.873 , precision of 0.868 , recall of 0.854 , and F1-score of 0.861 .
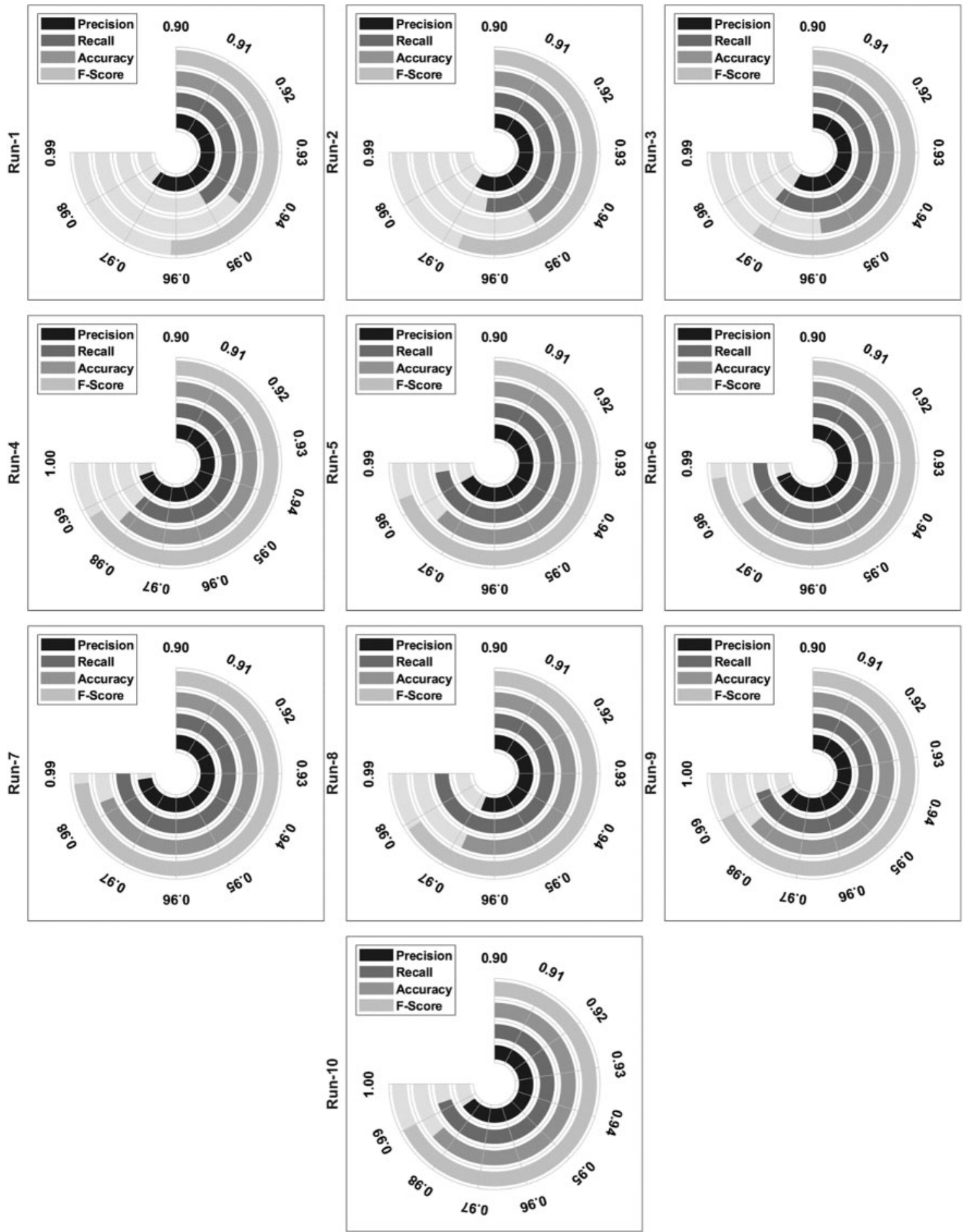

Figure 4: Result analysis of IoTDL-SIS method with different measures 
Table 2: Comparative analysis of IoTDL-SIS model with existing different states

\begin{tabular}{|c|c|c|c|c|}
\hline Methods & Accuracy & Precision & Recall & F1-score \\
\hline \multicolumn{5}{|c|}{ Existing techniques } \\
\hline \multicolumn{5}{|c|}{ Dataset-1 (70:30) Training and Testing } \\
\hline Naive Bayes & 0.835 & 0.827 & 0.81 & 0.818 \\
\hline DT (C4.5) & 0.857 & 0.851 & 0.838 & 0.845 \\
\hline SVM & 0.873 & 0.868 & 0.854 & 0.861 \\
\hline \multicolumn{5}{|c|}{ Dataset-1 (5-Fold CV) } \\
\hline Naive Bayes & 0.836 & 0.828 & 0.81 & 0.819 \\
\hline DT (C4.5) & 0.858 & 0.852 & 0.839 & 0.845 \\
\hline SVM & 0.875 & 0.869 & 0.855 & 0.862 \\
\hline
\end{tabular}

Dataset-2 (70:30) Training and Testing

\begin{tabular}{lllll}
\hline Naive Bayes & 0.844 & 0.834 & 0.826 & 0.83 \\
DT (C4.5) & 0.862 & 0.858 & 0.839 & 0.848 \\
SVM & 0.881 & 0.874 & 0.865 & 0.87 \\
\hline
\end{tabular}

Dataset-2 (5-Fold CV)

\begin{tabular}{lllll}
\hline Naive Bayes & 0.845 & 0.834 & 0.827 & 0.831 \\
DT (C4.5) & 0.863 & 0.859 & 0.84 & 0.849 \\
SVM & 0.882 & 0.876 & 0.866 & 0.871 \\
\hline
\end{tabular}

Proposed Method

\begin{tabular}{lllll}
\hline IoTDL-SIS & 0.971 & 0.980 & 0.981 & 0.980 \\
\hline
\end{tabular}

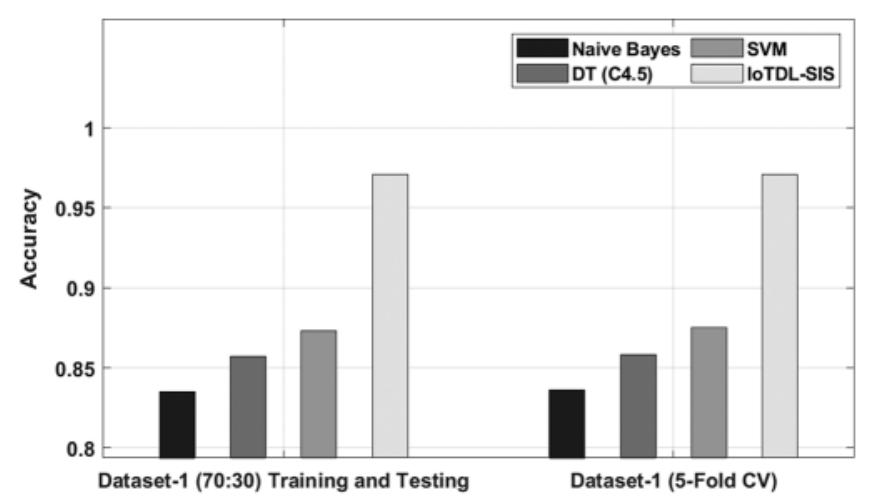

Figure 5: Accuracy analysis of IoTDL-SIS method in dataset 1 


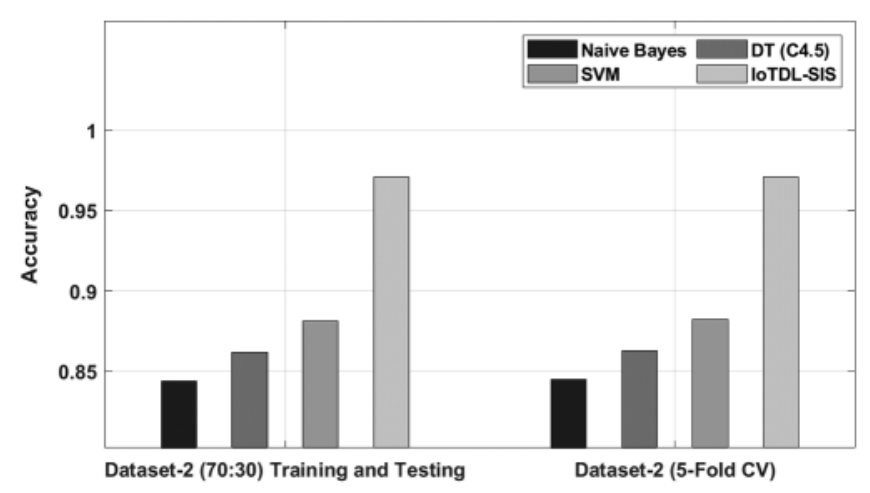

Figure 6: Accuracy analysis of IoTDL-SIS method in dataset 2

By investigating the performance of the IoTDL-SIS algorithm on the applied dataset-1 with (5-Fold CV), the NB method has demonstrated inferior results with minimum accuracy of 0.836 , precision of 0.828 , recall of 0.81 , and F1-score of 0.819 . Along with that, the DT (C4.5) algorithm has obtained somewhat enhanced outcomes with an accuracy of 0.858 , precision of 0.852 , recall of 0.839 , and F1-score of 0.845 . Afterward, the SVM model has showcased reasonable performance with an accuracy of 0.875 , precision of 0.869 , recall of 0.855 , and F1-score of 0.862 . By determining the performance of the IoTDL-SIS manner on the applied dataset-2 with (70:30) Training and Testing, the NB method has outperformed inferior outcome with the worse accuracy of 0.844 , precision of 0.834, recall of 0.826 , and F1-score of 0.83 . At the same time, the DT (C4.5) approach has obtained somewhat increased outcomes with an accuracy of 0.862 , precision of 0.858 , recall of 0.839 , and F1score of 0.848 . Then, the SVM model has depicted moderate performance with an accuracy of 0.881 , precision of 0.874 , recall of 0.865 , and F1-score of 0.87 .

By inspecting the performance of the IoTDL-SIS method on the applied dataset-2 with (5-Fold CV) Training and Testing, the NB algorithm has demonstrated inferior results with the minimum accuracy of 0.845 , precision of 0.834 , recall of 0.827 , and F1-score of 0.831 . In line with this, the DT (C4.5) approach has achieved somewhat increased outcomes with an accuracy of 0.863 , precision of 0.859 , recall of 0.84 , and F1-score of 0.849 . Afterward, the SVM technique has showcased moderate efficiency with an accuracy of 0.882 , precision of 0.876 , recall of 0.866 , and F1-score of 0.871 . However, the proposed IoTDL-SIS technique has been found to be superior to other techniques with the maximum accuracy of 0.971 , precision of 0.980 , recall of 0.981 , and F1-score of 0.980 .

\section{Conclusion}

In this study, a smart irrigation system using IoTDL-SIS technique is designed for effectual water utilization with less human interventions. The proposed IoTDL-SIS technique operates on three levels namely farmland, cloud server, and client level. The proposed IoTDL-SIS technique involves dissimilar sensors such as soil moisture, temperature, air temperature, and humidity for data acquisition purposes. The sensor data are transferred to the Arduino module which then transmits the sensor data to cloud server for further process. The cloud sever accomplishes the data analysis process by means of three different processes namely DSVM based regression, clustering, and AIOA-DBN based classification. A detailed experimental results analysis demonstrated the promising performance of presented method over the other recent state of art techniques interms of different models with 
the maximum accuracy of 0.971 . In future, the presented model can be realized in a smart phone environment to assist farmers instantly in real time.

Funding Statement: The authors extend their appreciation to the Deanship of Scientific Research at King Khalid University for funding this work under Grant Number (RGP1/53/42). www.kku.e du.sa. This research was funded by the Deanship of Scientific Research at Princess Nourah bint Abdulrahman University through the Fast-track Research Funding Program.

Conflicts of Interest: The authors declare that they have no conflicts of interest to report regarding the present study.

\section{References}

[1] M. S. Munir, I. S. Bajwa, M. A. Naeem and B. Ramzan, "Design and implementation of an iot system for smart energy consumption and smart irrigation in tunnel farming," Energies, vol. 11, no. 12, pp. 3427, 2018.

[2] T. Vaiyapuri, V. S. Parvathy, V. Manikandan, N. Krishnaraj, D. Gupta et al., "A novel hybrid optimization for cluster-based routing protocol in information-centric wireless sensor networks for iot based mobile edge computing," Wireless Personal Communications, 2021. In press, https://doi.org/10.1007/ s11277-021-08088-w.

[3] K. Shankar, E. Perumal, M. Elhoseny and P. Thanh Nguyen, "An iot-cloud based intelligent computeraided diagnosis of diabetic retinopathy stage classification using deep learning approach," Computers, Materials \& Continua, vol. 66, no. 2, pp. 1665-1680, 2021.

[4] H. Sattar, I. S. Bajwa, R. U. Amin, N. Sarwar, N. Jamil et al., "An iot-based intelligent wound monitoring system," IEEE Access, vol. 7, pp. 144500-144515, 2019.

[5] B. L. Nguyen, E. L. Lydia, M. Elhoseny, I. V. Pustokhina, D. A. Pustokhin et al., "Privacy preserving blockchain technique to achieve secure and reliable sharing of iot data," Computers, Materials \& Continua, vol. 65 , no. 1, pp. 87-107, 2020.

[6] M. Elhoseny, M. M. Selim and K. Shankar, "Optimal deep learning based convolution neural network for digital forensics face sketch synthesis in internet of things (iot)," International Journal of Machine Learning and Cybernetics, 2020. In press, https://doi.org/10.1007/s13042-020-01168-6.

[7] B. Sarwar, I. S. Bajwa, N. Jamil, S. Ramzan and N. Sarwar, "An intelligent fire warning application using iot and an adaptive neuro-fuzzy inference system," Sensors, vol. 19, no. 14, pp. 3150, 2019.

[8] I. V. Pustokhina, D. A. Pustokhin, T. Vaiyapuri, D. Gupta, S. Kumar et al., "An automated deep learning based anomaly detection in pedestrian walkways for vulnerable road users safety," Safety Science, vol. 142, pp. 105356, 2021.

[9] M. S. Munir, I. S. Bajwa, A. Ashraf, W. Anwar and R. Rashid, "Intelligent and smart irrigation system using edge computing and iot," Complexity, vol. 2021, pp. 1-16, 2021.

[10] R. T. Sanchez, H. N. Hellin, A. G. Frutos, R. S. Segundo, M. C. R. Abellón et al., "A decision support system for irrigation management: Analysis and implementation of different learning techniques," Water, vol. 12 , no. 2 , pp. $548,2020$.

[11] A. Bhoi, R. P. Nayak, S. K. Bhoi, S. Sethi, S. K. Panda et al., "IoT-iirs: Internet of things based intelligentirrigation recommendation system using machine learning approach for efficient water usage," PeerJ Computer Science, vol. 7, pp. e578, 2021.

[12] J. Yu, S. Tang, L. Zhangzhong, W. Zheng, L. Wang et al., "A deep learning approach for multi-depth soil water content prediction in summer maize growth period," IEEE Access, vol. 8, pp. 199097-199110, 2020.

[13] P. K. Kashyap, S. Kumar, A. Jaiswal, M. Prasad and A. H. Gandomi, "Towards precision agriculture: Iotenabled intelligent irrigation systems using deep learning neural network," IEEE Sensors Journal, vol. 21, no. 16, pp. 1-1, 2021. 
[14] S. Akshay and T. K. Ramesh, "Efficient machine learning algorithm for smart irrigation," in 2020 Int. Conf. on Communication and Signal Processing (ICCSP), Chennai, India, pp. 867-870, 2020.

[15] M. E. Karar, M. F. Al-Rasheed, A. F. Al-Rasheed and O. Reyad, "Iot and neural network-based water pumping control system for smart irrigation," Information Sciences Letters, vol. 9, no. 2, pp. 107-112, 2020.

[16] R. S. Krishnan, E. G. Julie, Y. H. Robinson, S. Raja, R. Kumar et al., "Fuzzy logic based smart irrigation system using internet of things," Journal of Cleaner Production, vol. 252, pp. 119902, 2020.

[17] N. K. Nawandar and V. R. Satpute, "IoT based low cost and intelligent module for smart irrigation system," Computers and Electronics in Agriculture, vol. 162, pp. 979-990, 2019.

[18] Z. Qi, B. Wang, Y. Tian and P. Zhang, "When ensemble learning meets deep learning: A new deep support vector machine for classification," Knowledge-Based Systems, vol. 107, pp. 54-60, 2016.

[19] S. Ryu, J. Noh and H. Kim, "Deep neural network based demand side short term load forecasting," Energies, vol. 10, no. 1, pp. 3, 2016.

[20] A. Bagheri, M. Zandieh, I. Mahdavi and M. Yazdani, "An artificial immune algorithm for the flexible job-shop scheduling problem,” Future Generation Computer Systems, vol. 26, no. 4, pp. 533-541, 2010. 